Enferm Bras 2019;18(3):373-81

https://doi.org/10.33233/eb.v18i3.3086

\title{
ARTIGO ORIGINAL \\ Estadiamento do câncer de colo uterino em um hospital de referência
}

Fernanda Furtado da Cunha, M.Sc.*, Maria da Conceição Nascimento Pinheiro, D.Sc.**, Anderson Roberto de Sales Corrêa***

*Enfermeira assistente da Unidade de Assistência de Alta Complexidade em Oncologia (HUJBB), ${ }^{* \star}$ Professora titular do Núcleo de Medicina Tropical/UFPA, ${ }^{* \star \star}$ Especialista em Enfermagem Oncológica

Recebido em 7 de maio de 2018; aceito em 1 de maio de 2019.

Correspondência: Fernanda Furtado da Cunha, Tv. Dr. Enéas Pinheiro, 1700/903, 66095-105 Belém PA, E-mail: furtadof@yahoo.com.br; Maria da Conceição Nascimento Pinheiro: mconci@ufpa.br; Anderson Roberto de Sales Corrêa: anderson.sales13@gmail.com

\section{Resumo}

Introdução: O câncer de colo do útero apresenta um dos mais altos potenciais de cura quando diagnosticado e tratado em estágios iniciais ou fases precursoras. O objetivo deste estudo foi avaliar o estadiamento clínico do câncer de colo uterino na época do diagnóstico, em um hospital de referência para câncer no Estado do Pará no período de 2001 a 2010. Métodos: Trata-se de um estudo observacional, retrospectivo, descritivo e analítico. A população alvo da pesquisa envolveu mulheres com diagnóstico de câncer de colo do útero constante no Registro Hospitalar de Câncer. As variáveis utilizadas incluíram: idade, ocupação, procedência, grau de instrução, diagnóstico histopatológico, estadiamento, local do tumor primário e desfecho. Resultados: As mulheres chegaram à unidade de referência com estadiamentos clínicos avançados (IIB e IIIB), com o carcinoma invasor contribuindo com a maioria dos casos. Os estadiamentos mais frequentes foram IIB, IIIB e IB. Apesar das campanhas de incentivo à prevenção, a ocorrência do câncer de colo uterino no estádio invasor ainda é muito alta, em detrimento dos casos de carcinoma in situ, os quais foram menos frequentes. Conclusão: As mulheres chegaram à unidade de referência em estágios avançados do câncer, principalmente nos estadiamentos IIB e IIIB. O carcinoma invasor é o tipo mais frequente contribuindo com $81,4 \%$ e o adenocarcinoma invasor com $7,1 \%$ do total das lesões malignas do colo. A maior parte das mulheres era maior de 40 anos de idade, de baixa escolaridade e oriundas da região metropolitana de Belém.

Palavras-chave: neoplasias do colo do útero, prevenção \& controle, estadiamento de neoplasias, papillomaviridae.

\begin{abstract}
Staging of uterine cervical cancer in a reference hospital

Introduction: Cervical cancer presents one of the highest potentials of cure when diagnosed and treated in early stages or precursor stages. The objective of this study was to evaluate the clinical staging of cervical cancer at the time of diagnosis in a reference hospital for cancer in the State of Pará from 2001 to 2010. Methods: This is a retrospective, descriptive, observational and analytical study. The target population of the study involved women diagnosed with cervical cancer in the Hospital Registry of Cancer. The variables used included: age, occupation, origin, educational level, histopathological diagnosis, staging, primary tumor site and outcome. Results: Women arrived at the reference unit with advanced clinical staging (IIB and IIIB), with invasive carcinoma contributing most cases, followed by invasive adenocarcinoma. In addition to the uninformed cases, the most frequent staging was IIB, IIIB and IB. Despite prevention campaigns, the incidence of cervical cancer in the invasive stage is still very high, to the detriment of cases of carcinoma in situ, which were less frequent. Conclusion: Women reached the reference unit in the advanced stages of cancer, mainly in the IIB and IIIB stages. Invasive carcinoma is the most frequent type contributing $81.4 \%$ and invasive adenocarcinoma with $7.1 \%$ of all malignant lesions in the colon. Most of the women affected were over 40 years of age, of low schooling and coming from the metropolitan area of Belém.
\end{abstract}

Key-words: uterine cervical neoplasms, prevention \& control, neoplasm staging, papillomaviridae. 


\section{Resumen \\ Estadificación del cáncer de cuello uterino en un hospital de referencia}

Introducción: El cáncer de cuello de útero presenta uno de los más altos potencial de curación cuando se diagnostica y se trata en etapas iniciales o fases precursoras. El objetivo de este estudio fue evaluar la estadificación clínica del cáncer de cuello uterino en la época del diagnóstico, en un hospital de referencia para cáncer en el Estado de Pará en el período de 2001 a 2010. Métodos: Se trata de un estudio observacional, retrospectivo, descriptivo, y analítico. La población objetivo de la investigación involucró a mujeres con diagnóstico de cáncer de cuello de útero constante en el Registro Hospitalario de Cáncer. Las variables utilizadas incluyeron: edad, ocupación, procedencia, grado de instrucción, diagnóstico histopatológico, estadificación, lugar del tumor primario y desenlace. Resultados: Las mujeres llegaron a la unidad de referencia con estadios clínicos avanzados (IIB y IIIB), con el carcinoma invasor contribuyendo con la mayoría de los casos. Los estadios más frecuentes fueron IIB, IIIB e IB. A pesar de las campañas de incentivo a la prevención, la ocurrencia del cáncer de cuello uterino en el estadio invasor aún es muy alta, en detrimento de los casos de carcinoma in situ, los cuales fueron menos frecuentes. Conclusión: Las mujeres llegaron a la unidad de referencia en etapas avanzadas del cáncer, principalmente en los estadios IIB y IIIB. El carcinoma invasor es el tipo más frecuente contribuyendo con el $81,4 \%$ y el adenocarcinoma invasor con el $7,1 \%$ del total de las lesiones malignas del cuello. La mayor parte de las mujeres era mayor de 40 años de edad, de baja escolaridad y oriundas de la región metropolitana de Belém.

Palabras-clave: neoplasias del cuello uterino, prevención \& control, estadificación de neoplasias, papillomaviridae.

\section{Introdução}

O câncer do colo uterino é uma doença de evolução prolongada a partir de mudanças intraepiteliais que se transformam em lesões invasoras em um período médio de cinco a seis anos. A forma invasiva é precedida por uma longa fase pré-invasiva, identificada como neoplasia intraepitelial cervical (NIC), categorizada em graus I, II e III ou lesão de baixo grau (NIC I) e lesões de alto grau (NIC II e NIC III). As formas avançadas são classificadas pelos seus estadiamentos clínico e patológico segundo a classificação TNM [1]. Atualmente, a 8ª edição da classificação de tumores está em vigor, tendo o objetivo de estimar fatores prognósticos, estratificação clínica e fatores de risco. O modelo recomendado para estimar o estadiamento em população considerará o TNM e fatores prognósticos (clínico e patológico), leva em conta em geral: a presença ou não de invasão, a presença de doença macroscópica ou microscópica, profundidade de invasão do estroma, tamanho da lesão, invasão de estruturas adjacentes, comprometimento de linfonodos e presença ou não de metástases [2].

O câncer do colo uterino representa uma das principais causas de óbito na população feminina no Brasil, com elevadas taxas de incidência e progressivo da mortalidade proporcional para as mulheres brasileiras no período de 2005 a 2014, sendo observada significativa diferença entre as regiões brasileiras [3]. Sua incidência é maior em países menos desenvolvidos. Em geral, surge em mulheres a partir de 30 anos de idade, aumentando seu risco rapidamente até atingir o pico etário entre 50 e 60 anos. Segundo o Instituto Nacional do Câncer (INCA), o Brasil apresentou uma sobrevida para o câncer de colo do útero de aproximadamente $70 \%$, sendo mais incidente em Estados da Região Norte (23,97/100 mil habitantes), Centro-Oeste (20,72/100 mil habitantes) e Nordeste (19,49/100 mil habitantes) [1].

A linha de cuidados para câncer de colo uterino consiste na prevenção primária (prevenção do surgimento do câncer de colo uterino), prevenção secundária (detecção precoce por rastreio e tratamento de lesões potencialmente cancerígenas), prevenção terciária (tratamento do câncer de colo uterino para prevenir morbidade e mortalidade) e qualidade de vida (cuidados após o tratamento e suporte paliativo). Sendo a forma mais eficaz de controlar esse tipo de tumor diagnosticando e tratando as lesões precursoras e as lesões tumorais invasoras em seus estágios iniciais [4]. Programas de controle de câncer com níveis elevados de qualidade, cobertura e acompanhamento de mulheres com lesões precursoras identificadas podem reduzir a incidência do câncer cervical significativamente. O teste de alto risco para HPV vem demonstrando boa acurácia e de complementação diagnóstica e tratamento precoce. Entretanto, não se observa queda na mortalidade no Brasil, principalmente devido à dificuldade diagnóstica precoce dessa doença [3,5]. 
Na última década, o Pará é o estado da federação que têm registrado os maiores índices da doença, no país. Nesse contexto, conhecer a situação do estadiamento diagnóstico do câncer de colo entre as mulheres que estão dando entrada em um hospital de referência para câncer é de fundamental importância para o estabelecimento de medidas que visem à melhoria do programa de prevenção e controle voltado ao diagnóstico precoce dos estágios iniciais da doença e a redução das formas avançadas.

\section{Material e métodos}

Trata-se de um estudo observacional, retrospectivo, descritivo e analítico, que utilizou dados secundários oriundos do registro de câncer em um hospital de referência para câncer no estado do Pará para análise dos estádios do câncer de colo uterino por ocasião do diagnóstico.

Foram incluídos todos os casos confirmados de câncer de colo uterino através do exame histopatológico independentemente da idade e do estadiamento do tumor, e registrados no período de janeiro de 2001 a dezembro de 2010. Foram excluídos do estudo os casos cujas informações necessárias para a análise estavam incompletas e não constavam no registro. $O$ período escolhido neste estudo foi utilizado, visto a disponibilidade dos dados do registro hospitalar de câncer, dados posteriores ao ano de 2010 não foram coletados pelo serviço hospitalar durante a coleta de dados.

Os dados foram coletados diretamente do sistema de informações do hospital-Registro Hospitalar de Câncer (RHC). A coleta para identificação das pacientes deu-se através de código numérico, identificando a ordem e o ano que o dado foi registrado do sistema. As variáveis utilizadas incluíram para o estudo: idade, ocupação, procedência, grau de instrução, diagnóstico histopatológico, estadiamento, local do tumor primário e desfecho. A distribuição dos dados de acordo com a procedência foi realizada de acordo com as regionais de saúde adotada pela Secretaria Estadual de Saúde do Estado do Pará [6].

O software Statistical Package for the Social Sciences (SPSS) versão 15.0 foi utilizado para a confecção do banco de dados e análises estatísticas. As variáveis categóricas foram avaliadas pela estatística descritiva e, para comparação intragrupos, utilizou-se o teste do Quiquadrado de aderência $(p<0,05)$.

A pesquisa foi submetida ao Comitê de Ética em Pesquisa do Núcleo de Medicina Tropical da Universidade Federal do Pará, tendo como instituição coparticipante o Hospital Ophir Loyola, de acordo com o observado na portaria 466/12 do Conselho Nacional de Saúde que estabelece critérios para o estudo em seres humanos. Por se tratar de uma pesquisa com dados secundários a partir do Sistema de Informação Hospitalar, o presente estudo dispensou o uso de Termo de Consentimento Livre e Esclarecido (TCLE). Para tanto foi utilizado o Termo de Compromisso para Utilização de Dados e Prontuários (TCUD). Ressalta-se que o sistema utilizado não identifica as pacientes, portanto, foram assegurados o anonimato e a privacidade das informações obtidas.

\section{Resultados}

As faixas etárias de maior acometimento por câncer de colo, neste estudo, foram de 40 a 49 anos $(27,7 \%)$ e de 50 a 59 anos $(22,2 \%)$. Mulheres com ensino fundamental incompleto constituíram $48,2 \%$ da população estudada. A principal categoria ocupacional incluiu aposentadas/pensionistas, com 56,3\% (Tabela I).

A procedência dos casos com câncer de colo foi oriunda principalmente de municípios que compõem a $1^{\underline{a}}$ regional de saúde, com 2.352 casos $(39,9 \%)$, seguido da $11^{\underline{a}}$ regional com $525(8,9 \%)$ e $4^{a}$ regional com $429(7,3 \%)$ dos casos. A menor frequência observada foi oriunda da $10^{\underline{a}}$ regional, com 127 casos (2,16\%) (Figura I). 
Tabela I - Caracterização sociodemográfica de mulheres atendidas em hospital de referência para tratamento de câncer de colo do útero em Belém, Pará, 2001-2010.

\begin{tabular}{|c|c|c|c|}
\hline Variável & $\mathrm{n}$ & $\%$ & p-valor \\
\hline \multicolumn{4}{|l|}{ Idade (anos) } \\
\hline$<20$ & 6 & 0,1 & \multirow{10}{*}{$<0.0001^{*}$} \\
\hline $20-29$ & 284 & 4,8 & \\
\hline $30-39$ & 1090 & 18,5 & \\
\hline $40-49$ & 1633 & 27,7 & \\
\hline $50-59$ & 1312 & 22,2 & \\
\hline $60-69$ & 926 & 15,7 & \\
\hline $70-79$ & 474 & 8 & \\
\hline $80-89$ & 139 & 2,4 & \\
\hline$\geq 90$ & 35 & 0,6 & \\
\hline Total & 5899 & 100 & \\
\hline \multicolumn{4}{|l|}{ Instrução } \\
\hline Analfabeto & 1145 & 19,4 & \multirow{8}{*}{$<0.0001^{*}$} \\
\hline Fundamental incompleto & 2842 & 48,2 & \\
\hline Fundamental completo & 624 & 10,6 & \\
\hline Nível médio & 544 & 9,2 & \\
\hline Superior incompleto & 4 & 0,1 & \\
\hline Superior completo & 62 & 1,1 & \\
\hline Sem informação & 678 & 11,5 & \\
\hline Total & 5899 & 100 & \\
\hline \multicolumn{4}{|l|}{ Ocupação } \\
\hline Aposentada/pensionista & 3319 & 56,3 & \multirow{19}{*}{$<0.0001^{*}$} \\
\hline Comerciantes & 52 & 0,9 & \\
\hline Costureira & 87 & 1,5 & \\
\hline Cozinheiras/assemelhados & 37 & 0,6 & \\
\hline Funcionários públicos superiores & 11 & 0,2 & \\
\hline Pescadora & 26 & 0,4 & \\
\hline Professora & 139 & 2,4 & \\
\hline Técnicos e auxiliares & 90 & 1,5 & \\
\hline Trabalhador autônomo & 20 & 0,3 & \\
\hline Trabalhador de atividade braçal & 17 & 0,3 & \\
\hline Trabalhador de atividades rurais & 552 & 9,4 & \\
\hline Trabalhadores de serviços gerais & 911 & 15,4 & \\
\hline Trabalhadores de estética & 41 & 0,7 & \\
\hline Turismo e entretenimento & 15 & 0,3 & \\
\hline Vendedora & 82 & 1,4 & \\
\hline Outros & 27 & 0,5 & \\
\hline Não se aplica & 319 & 5,4 & \\
\hline Sem informação & 154 & 2,6 & \\
\hline Total & 5899 & 100 & \\
\hline
\end{tabular}

Fonte: Dados da pesquisa, 2016. 


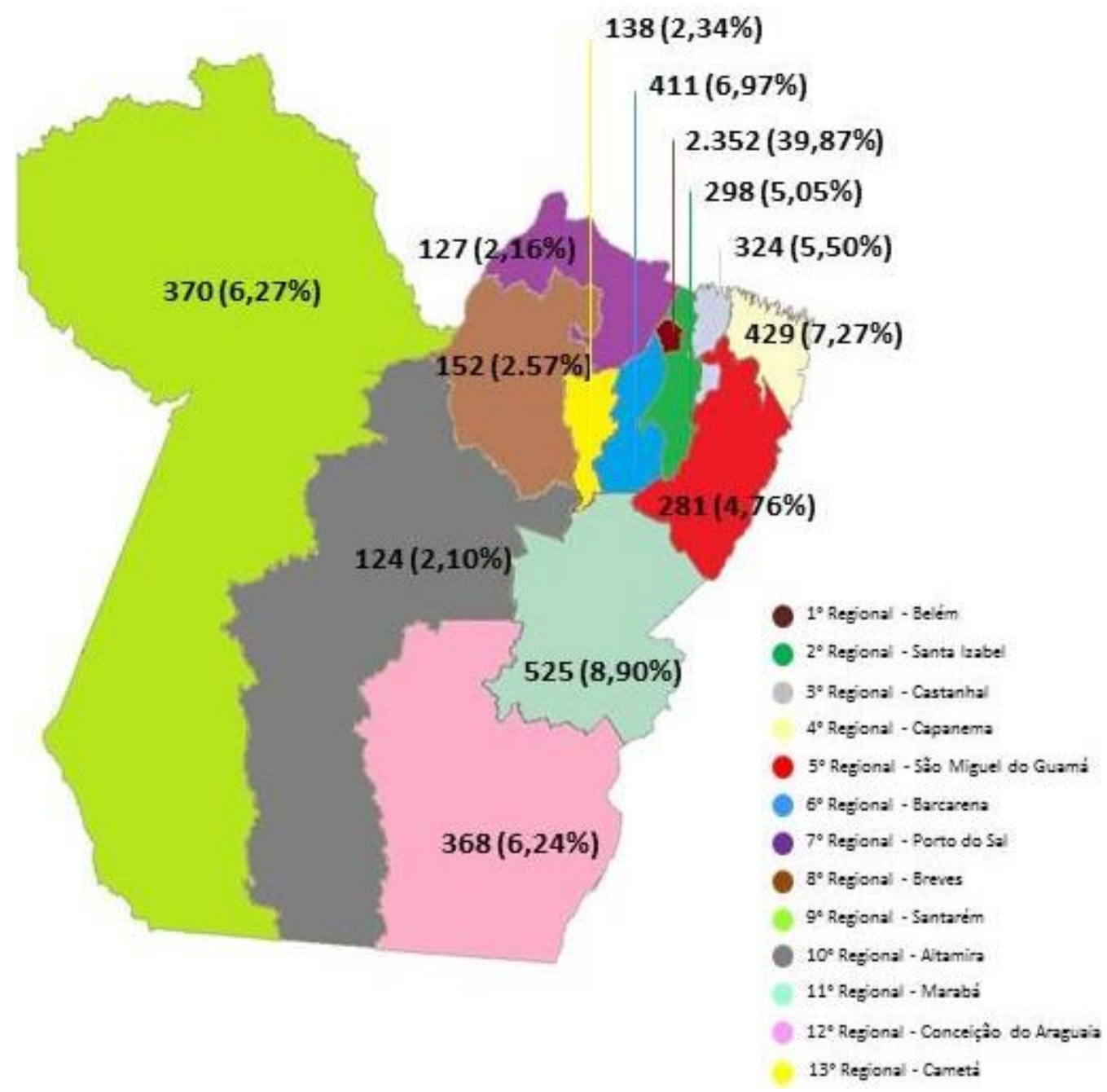

Fonte: Dados da pesquisa, 2016.

Figura 1 - Número absoluto e relativo de casos de câncer de colo de útero segundo a procedência, atendidos em hospital de referência em Belém/PA, 2001-2010.

O tipo histológico de maior frequência encontrado foi o carcinoma invasor, alcançando 2.961 casos na cérvix, sem origem especificada $(81,44 \%), 1.250$ casos na ectocérvix $(89,69 \%)$, 451 na endocérvix (66,52\%) e 162 em lesão sobreposta (84,9\%) (Tabela II).

Tabela II - Distribuição de câncer de colo de útero segundo a localização do tumor primário em mulheres atendidas em hospital de referência em Belém/PA, 2001-2010.

\begin{tabular}{lccccc}
\hline \multicolumn{1}{c}{ Tipo histológico } & Cérvix (SOE) & Ectocérvix & Endocérvix & Lesão sobreposta \\
\hline Carcinoma "in situ" & 332 & 50 & 24 & 3 \\
& $(9,14 \%)$ & $(3,59 \%)$ & $(3,54 \%)$ & $(1,58 \%)$ \\
Carcinoma invasor & 2961 & 1250 & 451 & 162 \\
& $(81,44 \%)$ & $(89,68 \%)$ & $(66,52 \%)$ & $(84,81 \%)$ \\
Adenocarcinoma "in situ" & 2 & - & 2 & - \\
& $(0,05 \%)$ & & $(0,29 \%)$ & \\
Adenocarcinoma invasor & 149 & 69 & 180 & 21 \\
& $(4,09 \%)$ & $(4,95 \%)$ & $(26,55 \%)$ & $(10,99 \%)$ \\
Neoplasia maligna & 36 & 5 & 8 & 2 \\
& $(0,99 \%)$ & $(0,35 \%)$ & $(1,18 \%)$ & $(1,04 \%)$ \\
NIC III & 156 & 20 & 13 & 3 \\
& $(4,29 \%)$ & $(1,43 \%)$ & $(1,92 \%)$ & $(1,58 \%)$ \\
Total & 3636 & 1394 & 678 & 191 \\
& $(100,00 \%)$ & $(100,00 \%)$ & $(100,00 \%)$ & $(100,00 \%)$ \\
\hline Fonte: Dados da pesquisa, 2016. & \multicolumn{3}{c}{}
\end{tabular}

Fonte: Dados da pesquisa, 2016. 


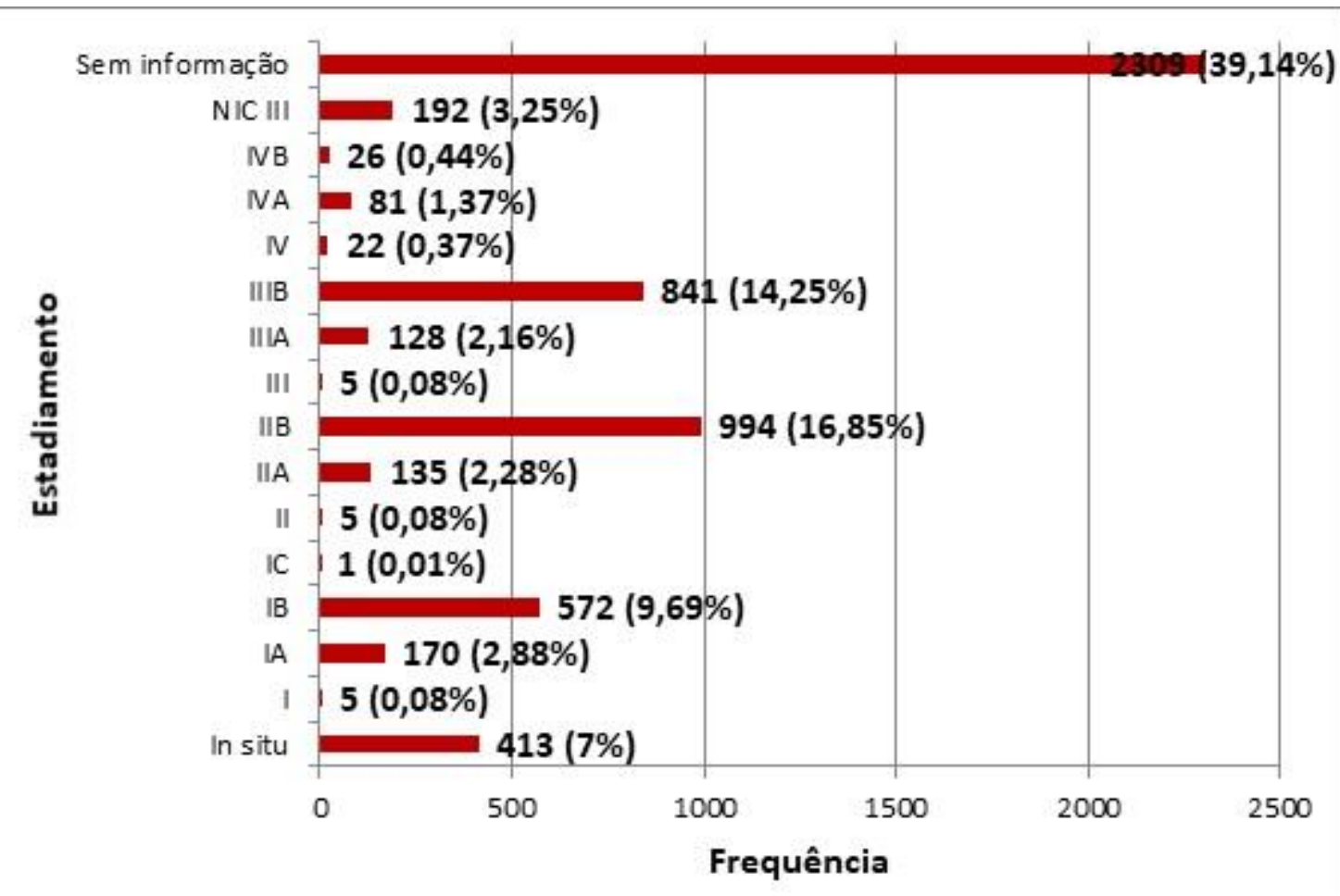

Fonte: Dados da pesquisa, 2016.

Figura II - Distribuição de estadiamento em mulheres atendidas em hospital de referência em Belém/PA 2001-2010.

Os indivíduos sem informação quanto ao estadiamento representaram 2.309(39,14\%), seguido dos casos com estágio IIB (16,85\%), estágio IIIB (14,25\%) e estágio IB (9,69\%) (figura II).

Em relação ao desfecho final do primeiro tratamento, 2.159 (36,60 \%) permaneceram com doença estável, seguido de $1.317(22,3 \%)$ casos que não realizaram nenhum tratamento. Aqueles com a doença em progressão contabilizaram 551(9,30\%) casos (figura III).

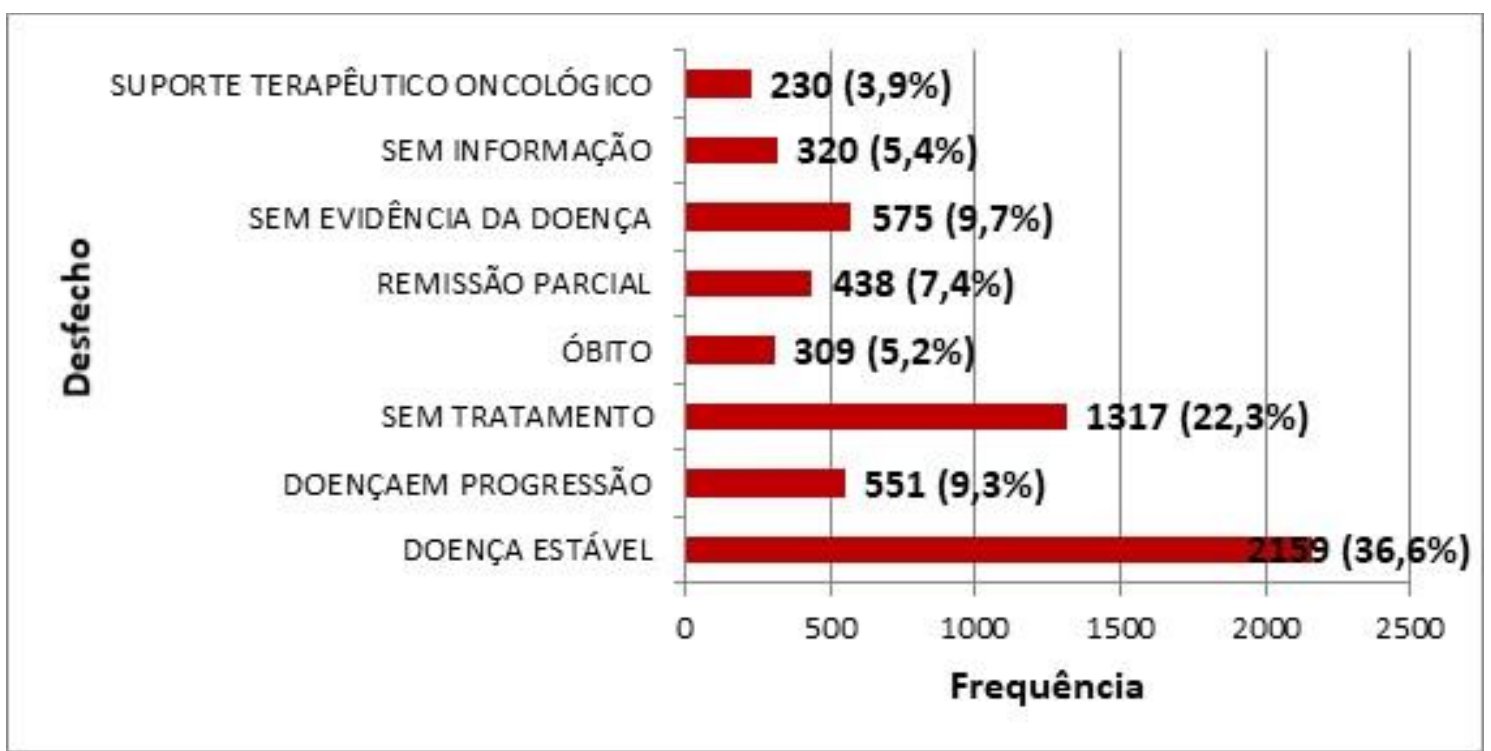

Fonte: Dados da pesquisa, 2016.

Figura III - Desfecho de tratamento para câncer de colo de útero em mulheres atendidas em hospital de referência em Belém/PR, 2001-2010. 
Neste estudo, observou-se que a incidência de câncer de colo uterino teve crescimento a partir da faixa etária de 30 a 39 anos, concentrando cerca de $50 \%$ dos casos entre 40 e 59 anos de idade. Isto reforça a importância do início precoce e da periodicidade do exame preventivo do câncer, conforme recomendado pelo programa de prevenção do câncer do colo uterino. De acordo com esse programa, a prevenção através do exame de Papanicolau deve iniciar na segunda década de vida com vista ao diagnóstico e tratamento precoce das lesões. A idade é um elemento fortemente associado à doença.

Na fase mais jovem, a mulher está mais exposta às doenças sexualmente transmissíveis, incluindo HPV, o principal agente causador do câncer de colo uterino. A frequência maior das fases avançadas em mulheres na idade madura ou idosa reflete o longo período do processo evolutivo em que as lesões precursoras não foram detectadas. Estudos de série histórica realizados no Brasil demonstraram que a tendência de casos em pacientes idosas, com 80 anos ou mais, aumentou, reforçando a hipótese de exposição à carcinógenos e mutações que promovem o câncer. Análises de acompanhamento citológicas foram realizadas em grupos de mulheres com diagnóstico citológico anormal, sendo identificado que o diagnóstico de atipia em células glandulares demonstrou maior risco para progressão da doença cervical, e mulheres acometidas com idade inferior a 25 anos obtiveram melhor taxa de resposta para regressão das lesões. Inferindo-se o importante papel imunológico da mulher jovem na regressão das lesões $[7,8]$.

A baixa escolaridade foi outro fator importante em associação com o câncer, com 48,2\% com ensino fundamental incompleto. A falta de instrução ou baixo nível de instrução dificulta a compreensão e a adoção das medidas educativas no combate ao câncer. Alguns estudos têm chamado atenção para a associação da baixa escolaridade com as maiores prevalências e maiores taxas de mortalidade por câncer do colo do útero. Em estudo realizado de dados secundários do INCA e DATASUS foi observado que: ao se analisar o aspecto sociodemográfico, a escolaridade apresentou a maior significância na incidência do câncer de colo uterino, com mulheres com nenhum a três anos de escolaridade, representando $84,17 \%$ da amostra populacional nesta revisão [9]. A educação é uma estratégia fundamental, mesmo produzindo resultados em médio e longo prazo, e que precisa ser constantemente utilizada para se reduzir as desigualdades sociais e, consequentemente, as taxas de mortalidade pelo câncer do colo do útero [9].

Cerca de $40 \%$ dos casos na presente pesquisa foram procedentes de municípios pertencentes a 1a regional de saúde paraense, da qual faz parte a região metropolitana de Belém e municípios próximos. Isto pode estar relacionado ao acesso aos serviços de saúde, devido à proximidade com os centros de atenção secundária e terciária, os quais estão mais concentrados nos centros urbanos. Estes achados são essenciais para os gestores dos Sistemas de Saúde Estadual e Municipal que devem se planejar para fornecer a essas mulheres, além do transporte para o tratamento fora do domicílio, as condições necessárias para o tratamento em tempo oportuno, aumentando suas possibilidades de recuperação e cura ou, de outra forma, a descentralização de atendimento referencial da região metropolitana para os demais pontos do Estado.

A localização do câncer invasivo sem origem especificada não permite determinar com precisão sua origem ou tempo de evolução. O tempo médio estimado para que uma lesão intraepitelial seja detectada antes de se tornar invasora é de 10 a 12 anos. O câncer de colo uterino surge na região escamocolunar, podendo desenvolver um padrão de células escamosas acometendo, principalmente, a ectocérvix. Provavelmente, a alta frequência do tumor primário sem especificação seja composta na maioria, por lesão inicial na ectocérvix. De outra forma, a neoplasia pode assumir um padrão glandular se desenvolvendo na endocervix. Em termos de ocorrência, o adenocarcinoma é menos frequente do que o tipo escamoso. Os tipos que crescem na endocervix desenvolvem geralmente um padrão de adenocarcinoma $[10,11]$.

Estudo realizado no tocante ao rastreamento nas últimas décadas demonstrou que o ao ser efetivamente aplicado, há uma progressiva redução de novos casos e aumento na proporção dos estádios iniciais de câncer do colo uterino, contando com mais da metade dos tumores invasores detectados ainda em estádio I [11]. Estas observações diferem da realidade encontrada em nosso estudo, em que a maior parte das mulheres chegam ao estágio II, seguido do estágio III, sugerindo que essas mulheres não foram cobertas pelo programa de rastreamento, e não realizaram o exame preventivo de acordo com as recomendações do programa de controle, estratégia importante na prevenção da fase avançada da doença. O diagnóstico precoce somado 
ao tratamento reduz, quase que totalmente, o desenvolvimento da doença, levando em conta que sua evolução é lenta e gradativa. Apesar de um grande número de mulheres ainda chegarem às unidades de referência com câncer do colo do útero, em estadiamento avançado, esse número vem diminuindo nos últimos anos, no país, porém, ainda está longe de alcançar os parâmetros de países desenvolvidos [1,11].

Os números absolutos mostram que, em 2002, morreram 4.091 mulheres por causa da enfermidade no Brasil. Dados epidemiológicos do INCA demonstram que o câncer de colo uterino é a terceira localização primária de incidência e de mortalidade, excluindo o câncer de pele não melanoma. No ano de 2016 foram estimados 5.847 óbitos para esta neoplasia, representando mortalidade de 4,70 óbitos para cada 100 mil mulheres. Provavelmente o crescimento em números absolutos se deve ao aumento da população feminina, no mesmo período. A incidência estimada e mortalidade no Brasil assemelham-se a outros países em desenvolvimento, porém há grande disparidade entre países já desenvolvidos e com programa de rastreamento precoce bem estruturado. Durante análise de mortalidade por região, o Norte ainda se destaca como o mais incidente na região com 23,97 casos por 100 mil habitantes, a região apresenta ainda as maiores mortalidades de todo o País no período de 2016, representando 5,71 por 100 mil mulheres, com números absolutos de 1.208 casos notificados de óbito por câncer de colo uterino, dados que corroboram a presente pesquisa, indicando o elevado estadiamento na ocasião diagnóstica e falha dos programas de rastreamento no serviço público [12].

Espera-se que a vacinação contra HPV no Brasil possa registrar a primeira e demais gerações de mulheres livres deste tipo de câncer. A medida, tomada em mais 100 países, pode fazer com que a atual geração tenha uma incidência significativamente menor da doença, já que existe a vacina contra um dos dois dos vírus responsáveis por $70 \%$ dos casos de câncer de colo de útero [13].

Esses dados refletem a importância e necessidade de acesso maior à informação, e o seguimento de curso da urbanização. O câncer está presente em todos os países, mas em cada um deles a doença apresenta um perfil diferenciado. Dependendo do local geográfico, o perfil do câncer pode assemelhar-se ao de países desenvolvidos e subdesenvolvidos. Apesar das políticas de prevenção, a região norte do Brasil continua em primeiro lugar em incidência de câncer do colo uterino, superando o câncer de mama, o que leva a crer uma falha na cobertura dos programas de prevenção e acesso a saúde.

Conclusão

Os resultados deste estudo revelaram que as mulheres chegaram à unidade de referência em estágios avançados do câncer, predominando os estadiamentos IIB e IIIB. O carcinoma invasor é o tipo mais frequente contribuindo com $81,4 \%$ e o adenocarcinoma invasor com $7,1 \%$ do total das lesões malignas do colo.

A maior parte das mulheres acometidas era maior de 40 anos de idade, de baixa escolaridade e oriundas da região metropolitana de Belém.

Apesar das campanhas de incentivo à prevenção, a ocorrência do câncer de colo uterino no estádio invasor ainda é muito alta. Além disso, observou-se diminuição dos casos insitu, podendo se concluir que as ações adotadas estão sendo ineficientes. Campanhas educacionais devem ser fortalecidas e novos estudos devem ser conduzidos na expectativa de identificar fatores que estejam interferindo no desempenho do programa de controle.

1. Instituto Nacional de Câncer. Brasil. Estimativa 2016/2017: incidência de câncer no Brasil. Rio de Janeiro: INCA; 2016.

2. American Joint Committee on Cancer. AJCC Cancer staging manual. 8nd. New-York: Springer; 2016.

3. Ceolin R, Nasi C, Paz AA, Linch GFC. Perfil de mortalidade por câncer de colo de útero no período de 2005-2014. Rev RECOM 2018; 8 (e1806). https://doi.org/10.19175/recom.v7i0.1806

4. Finocchario-Kessler S, Maloba M, Mabachi N, Ndikum-Moffor F, Bukusi E. Cervical cancer prevention and treatment research in Africa: a systematic review from a public health perspective. BMC Womens Health 2016;16(29):1-25.

https://doi.org/10.1136/bmi.k4823 
5. Arbyn M, Smith SB, Temin S, Sultana F, Castle P. Detecting cervical cancer and reaching underscreened women by using HPV testing on self samples: updated metaanalyses. BMJ Clinical Research 2018;363(k4823):1-21. https://doi.org/10.1136/bmi.k4823

6. Secretaria Estadual de Saúde (SESPA). 2017. [citado 2017 Dez 15]. Disponível em: http://www. saude.pa.gov.br/?page $\mathrm{id}=41$

7. Cech LR. Tendência de mortalidade por câncer de colo do útero no estado do Maranhão no período de 1994 a 2014 [monografia]. São Luís: Universidade Federal do Maranhão; 2017.

8. Santos AV. Rastreamento citológico: progressão e regressão de lesões cervicais [Dissertação]. Porto Alegre: Universidade Federal de Ciências da Saúde de Porto Alegre; 2017.

9. Cavalcante A, Simão G, Camargo I, Faria L, Moreira M, Álvares W. Relação da escolaridade com o número de mortes por câncer de colo uterino. ANAIS da $6^{\underline{a}}$ amostra de saúde 2014;2(1): 924.

10. Carvalho LP, Walois VSS, Silva IP, Melo TMS. Câncer cervical: relação entre HPV e co-fatores no desdobramento da oncogênese. Rev Cient FASETE 2018;15(1):297-315.

11. Instituto Nacional de Câncer. Brasil. Diretrizes brasileiras para o rastreamento do câncer do colo do útero. Rio de Janeiro: INCA; 2016.

12. Instituto Nacional de Câncer. Brasil. Atlas da mortalidade. Rio de Janeiro: INCA; 2019.

13. Gama DON, Silva MM, Carvalho RNC. Papiloma vírus humano: uma abordagem sobre prevenção e assistência. Rev Cient FASETE 2018;20(1):109-24. 